\title{
Principios y subprincipios para un currículo praxiológico de educación física
}

\author{
Raúl Martínez-Santos ${ }^{1}$ \\ Astrid Aracama ${ }^{1}$ \\ María P. Founaud ${ }^{2}$ \\ Asier Oiarbide ${ }^{1}$
}

\section{RESUMEN}

La acción motriz es la punta de lanza de la educación física, dice Parlebas, pero ¿qué posibilidad cabe de transformar esta declaración de principios en un currículo que oriente la reflexión pedagógica, la organización didáctica y la acción motriz de los distintos agentes escolares? Antes de avanzar hacia una nueva reforma legal, nos atrevemos a compartir los frutos de nuestro estudio y nuestros debates, tras identificar las tres cuestiones fundamentales de la educación física escolar e interpretarlas en clave praxiológica: la cátedra libre es, en realidad una cátedra cautiva por los derechos a la educación a la enseñanza que, en una sociedad abierta y democrática, fija los fines y los medios de enseñanza que limitan y orientan las decisiones docentes; la institución escolar genera una (in)compatibilidad lúdica que transforma la naturaleza del juego sin impedir, empero, que sean un medio de intervención formidable para la educación de las conductas de reproducción, de decisión, de convivencia y de expresión; por último, los efectos obtenidos por la educación física son transformaciones de las competencias motrices cuya evaluación práxica se puede concebir como una interpretación de signos situados.

Palabras Clave: Educación física escolar. Innovación pedagógica. Pragmatismo. Libertad de cátedra. Juego. Evaluación. Ludomotricidad

\footnotetext{
${ }^{1}$ Universidad del País Vasco.

2 Universidad de Zaragoza.
}

\section{Correspondência:}

Raúl Martínez-Santos. UPV/EHU, Facultad de Educación y Deporte, Portal de Lasarte, 71, 01007 Vitoria-Gasteiz, España, Email: raul.martinezdesantos@ehu.es 


\section{Princípios e subprincípios para um currículo praxiológico de educação física}

\section{RESUMO}

A ação motora é a ponta da lança da educação física, diz Parlebas, mas que possibilidade existe para transformar essa declaração de princípios em um currículo que norteie a reflexão pedagógica, a organização didática e a ação motriz dos diferentes agentes da escola? Antes de avançar para uma nova reforma legal, ousamos compartilhar os frutos de nosso estudo e de nossos debates, depois de identificar as três questões chave da educação física escolar e interpretá-las em uma chave praxiológica: a cadeira livre é uma cadeira cativa por o direito à educação e à ensino que, em uma sociedade democrática, define os objetivos e os meios que limitam e orientam as decisões educacionais; a instituição escolar gera uma (in)compatibilidade lúdica que transforma a natureza do jogo sem, no entanto, impedir que seja um meio formidável de intervenção para a educação da conduta de reprodução, decisão, convivência e expressão; finalmente, os efeitos obtidos pela educação física são transformações das habilidades motoras cuja avaliação práxica pode ser concebida como uma interpretação dos signos situados.

Palavras-chave: Educação física escolar. Inovação pedagógica. Pragmatismo. Liberdade acadêmica. Jogo. Avaliação. Ludomotricidade.

\section{Principles and subprinciples for a praxeological curriculum of physical education}

\section{ABSTRACT}

Motor action is the spearhead of physical education, says Parlebas, but what is the possibility of transforming this declaration of principles into a curriculum that guides the pedagogical reflection, the didactic organization and the motor action of the different school agents? Before moving towards a new legal reform, we dare to share the fruits of our study and our debates, after identifying the three fundamental questions of school physical education and interpreting them in a praxiological key: academic freedom is, in reality, an academic captivity in an open and democratic society, the rights to education and teaching set the aims and means of instruction that limit and guide teaching decisions; the school institution generates a playful (in)compatibility that transforms the nature of the game playing without impeding it to be a formidable intervention means for the education of conducts of reproduction, decision, coexistence and expression; finally, the effects obtained by physical education are transformations of motor competencies whose praxic evaluation can be conceived as an interpretation of situated signs.

Keywords: School physical education. Pedagogic innovation. Pragmatism. Academic freedom. Play. Evaluation. Ludomotricity. 


\section{DE MUSAS Y TEATROS}

Como el teatro, la educación física parece gozar de una mala salud de hierro, y su naturaleza parece ser, como la del teatro, la de un estado de crisis interminable en el que lo único que permanece es el cambio: una crisis tan arraigada que su manifestación más sobresaliente es la creatividad de sus agentes, como si de actores y escritores de teatro se tratara. Cabe la terrible posibilidad de que el desmenuzamiento que Parlebas (1967: 2017, p. 73 y ss.) detectó hace más de cincuenta años en la educación física francesa no fuera circunstancial, sino estructural, y que la crisis de las educaciones corporales anunciada por During (1981: 1992) fuera más un aviso a navegantes que un parte de defunción.

Este ensayo colectivo es el resultado de un experimento tan imaginario como imaginativo, surgido en medio de apasionados debates sobre la educación física, la praxiología y las ofertas públicas de empleo. Por darle un toque lúdico-dramático, podríamos plantearlo así:

Hemos recibido el encargo de escribir una obra original para ser estrenada después del verano. Se nos ha dado patente de corso, mínimas indicaciones y varias advertencias: la obra será representada en infinidad de ocasiones y lugares, por actores y actrices de todo pelaje y condición, con niveles de compromiso, entusiasmo y capacidad variables; los medios a disposición de los correspondientes elencos y direcciones artísticas no se esperan óptimos, pero sí suficientes; las tradiciones estéticas y culturales podrán provocar incomprensiones, rechazos y hasta adaptaciones que alteren sustancialmente el sentido original de nuestro trabajo; hasta cabe la posibilidad de que sea abandonada en un rincón por quienes la reciben como una imposición de quien no entiende los problemas que los comediantes de barro y barrio deben afrontar cada día para sacar adelante un arte con gajes demasiado pobres..

De inmediato, correspondiendo a la temeridad ajena con intrepidez y coraje, hemos aceptado el reto de enfrentarnos a una cuestión tan legitima como estremecedora: ¿es posible una educación física escolar praxiológica de cabo a rabo? Estamos seguros de que sí, pero ¿disponemos de suficientes recursos conceptuales para levantar al menos el armazón del edificio? $Y$ ante semejante envite, ¿seremos capaces de repensar de forma operativa y comunicable el área de educación física desde la raíz?

La educación física básica, el área de educación física de la educación básica, es una de las piezas del derecho positivo, del conjunto de leyes vigentes en un momento dado. Por esta razón, se puede decir que nuestro reto consiste en dar forma a una de esas piezas que, en esencia, es un "ámbito óntico-práctico" (ROBLES, 1984, p. 72), un conjuntos de competencias jurídicas o posibilidades de 
acción "por parte de los sujetos de las decisiones inmanentes y de los sujetos de las decisiones dirimentes, posibilidades que al propio tiempo son expresiones de la acción necesaria".

La única profesión regulada en nuestro campo laboral es la docente, en sus distintos niveles: primaria, secundaria, bachillerato, formación profesional y enseñanzas especiales, ya que su ejercicio pasa por la adquisición de unas competencias profesionales que den visos de realidad al compromiso de permitir el desarrollo de las competencias previstas en las normativas: las competencias motrices. Como muy bien saben nuestros colegas de patio y claustro, su actividad es susceptible de ser inspeccionada por los "sujetos de las decisiones dirimentes" de Robles.

Nuestro punto de partida no es ningún secreto. Hemos podido mostrar (MARTÍNEZ-SANTOS, En prensa) que la afirmación de Parlebas: "La acción motriz es la punta de lanza de la educación física", es la metáfora praxiológica perfecta, como queda ilustrado en la figura 1 . La acción motriz*3, la capacidad de acción puesta en juego en las situaciones ludomotrices, permite articular sobre el terreno los fines de cualquier propuesta educativa democrática con las exigencias jurídicas de una práctica profesional que haya pasado de la magia a la investigación científica (1969: PARLEBAS, 2017, p. 209).

La corriente de la acción motriz, por aprovechar la denominación empleada por Zoro (2002, p. 84-87), no es un método ni una moda, sino un sistema de ideas claras y creencias educadas, en el sentido que Peirce daba a las unas y las otras: un sistema de hábitos de acción, ya sea en forma de argumentos o rebates, diseños de investigación o diseños de intervención.

Si al jugar somos los indicios que engendramos, al especular somos los símbolos que nuestro pensamiento incorpora, ya sea en forma de concepto, argumento, diagrama o proyecto. La educación física escolar es un objeto jurídico que se materializa -se hace signo- en forma de documentos que, a su vez, se materializan -al interpretarse- en forma de prácticas motrices. A sensu contrario, cualquier situación motriz educativa es un signo del propio sistema que debe ser interpretado, valorado en tanto tal, en tanto que materialización de una serie de principios jurídicos, pedagógicos y didácticos.

Las situaciones de práctica motriz también son organizaciones significantes, y por eso precisamente debemos estar seguros de que la cadena de mando (ya llegará el momento de encontrar una imagen mejor...) es fuerte y consistente; debemos asegurar que los textos y los discursos se alinean de manera coherente de principio a fin, desde parlamento al patio del colegio.

\footnotetext{
3 Todos los términos señalados con un asterisco $\left(^{*}\right)$ remiten al léxico de praxiología motriz (PARLEBAS, 2001), cuya edición francesa puede ser consultada en la siguiente dirección: https://books.openedition.org/insep/1067.
} 


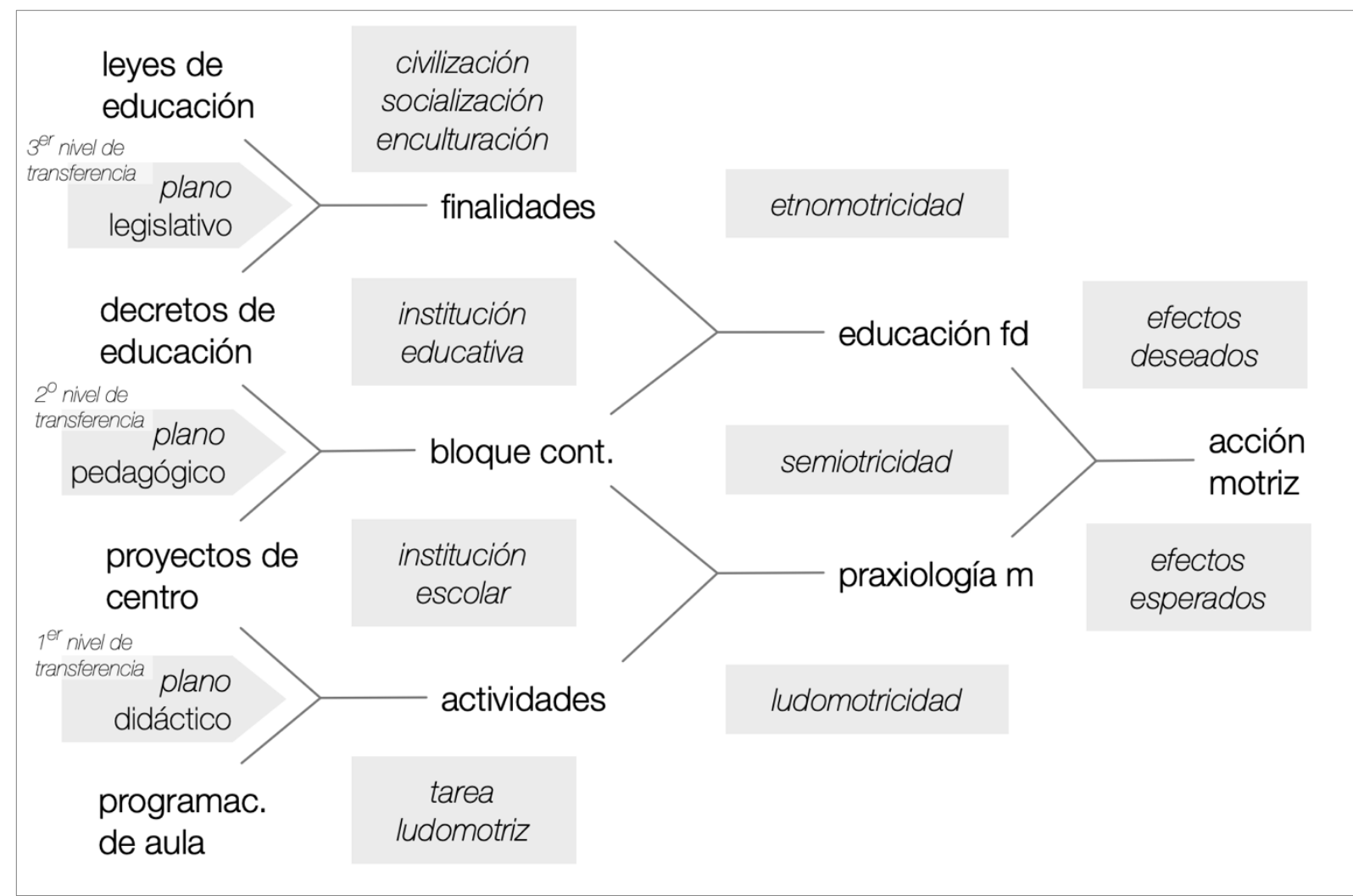

Figura 1 - Lógica interna de la educación física escolar praxiológica.

Goffman desarrolló gran parte de sus aportaciones a partir de la metáfora teatral de la vida social, de la que hace un uso extenso en La presentación de la persona en la vida cotidiana (1959: 1997). Es muy posible que, como apunta Manning (1991, p. 72), una "metáfora [sea] valiosa solo como un apéndice para la investigación en ciencias sociales: es la manivela para arrancar el motor, pero no el combustible para alimentar el automóvil. Las metáforas tienen vida propia, pero ¿no es eso, precisamente, lo que las hace un recurso privilegiado para el razonamiento más creativo? Arrastrados por las metáforas, nos adentramos en nuestro experimento como si de un juego de rol se tratara, intentando resolver el enigma que se nos ha planteado con la esperanza de que nuestra solución resulte, también, inspiradora para nuestros colegas.

Nuestra tesis es que las tres cuestiones fundamentales de la educación física curricular -a saber, la de los fines, la de los medios de enseñanza y la de los procedimientos de evaluación- se pueden formular y materializar alrededor del elemento clave de toda pedagogía: la acción. Los diagramas también son conceptos, decía Peirce, y creemos haber superado con éxito esta primera prueba de razonamiento icónico (Figura 1): ¿seremos igual de hábiles al pensar con las palabras? Con una pizca enorme de ingenio prestado; con la inspiración de Thalía, la festiva, y Terpsícore, la que deleita en la danza: en definitiva ¿seremos capaces de pasar de las musas de la praxiología motriz al teatro de las clases de educación física? 


\section{Principio de CÁtedRA CAUTIVA}

Como relata González del Valle (1981), la doctrina sobre la libertad de cátedra: "La ciencia y su docencia son libres", fue enunciada por primera vez en la Constitución Imperial alemana de 1849. Con el tiempo desarrolló un significado multidimensional: aseguramiento de libertad de opinión docente; una universidad al servicio de la ciencia, no del estado; aseguramiento de esta autonomía mediante financiación pública; aseguramiento de su desarrollo mediante una gestión académica universitaria que privilegie a los profesores cualificados (GONZÁLEZ DEL VALLE, 1981, p. 318). Llega a sorprender, por tanto, que la Constitución Española (C.E.) de 1978 la recoja en el articulo 20.1 -junto con los de expresión, creación, comunicación e información-, y que sólo quede vinculada de forma indirecta con el art 27, habida cuenta de su íntima relación con los derechos a la educación, a la enseñanza y a la autonomía universitaria recogidos en él.

Si Nando y Sanz (2019) están en lo cierto por lo que respecta al profesorado no universitario, y nada nos hace pensar lo contrario, la situación actual se caracteriza por falta de normativa especifica, ausencia de claridad conceptual y confusión entre la libertad de cátedra y la de enseñanza. Aun así, su aplicabilidad a la enseñanza no universitaria ya quedó aclarada tras la sentencia 5/1981 del Tribunal Constitucional4:

A la vista de los debates parlamentarios, que son un importante elemento de interpretación, aunque no lo determinen, [...] el constituyente de 1978 ha querido atribuir esta libertad a todos los docentes, sea cual fuere el nivel de enseñanza en el que actúen y la relación que media entre su docencia y su propia labor $(\S 9.2)$.

Lo que se debe entender tras este reconocimiento es que la libertad de enseñanza (art. 27 de la C.E.) implica para quienes la ejercen el derecho "a desarrollarla con libertad dentro de los límites propios del puesto docente que ocupan (art. $20.1 \mathrm{c}) "$ ( $\S 7.2$, cursivas añadidas por nosotros). El recordado Tomás y Valiente, en un voto particular secundado por otros tres magistrados, aportó un comentario interesante sobre la cuestión de los límites de la libertad de cátedra:

La libertad de cátedra está limitada por el respeto a los derechos contenidos en el Título I de la Constitución [sobre los derechos y deberes fundamentales] y a los preceptos de las Leyes que los desarrollen; lo cual obliga, en la medida en que el derecho a establecer un ideario educativo (art. 34.1 de la L.O.E.C.E.) es un complemento del derecho de los padres recogido en la Constitución (art. 27.3), a conectar libertad de cátedra e ideario educativo y a interpretar en este sentido los términos del art. 15

\footnotetext{
${ }^{4}$ Sentencia parcialmente estimatoria del recurso presentado por los grupos socialistas del Senado contra la Ley Orgánica 5/1980, de 19 de junio, por la que se regulaba el Estatuto de Centros Escolares (L.O.E.C.E.).
} 
de la L.O.E.C.E. Implica también el reconocimiento, como límite específico de aquella libertad, de "la protección de la juventud y de la infancia"; el grado de madurez personal de los destinatarios de la enseñanza y el nivel científico de los conocimientos que a ellos se han de transmitir condicionan la amplitud de la libertad que cada profesor puede ejercer: amplitud muy variable en relación con quienes enseñen en los centros escolares regulados por la Ley Orgánica 5/1980, ya que en ellos se comprenden desde los más elementales niveles de enseñanza hasta los cursos del Bachillerato y de Orientación universitaria, más cercanos ya a la enseñanza universitaria ( $\$ 13.1$, cursivas añadidas por nosotros).

Es posible que las limitaciones de esta deseada libertad sean menos debidas a su burocratización, a su ejercicio en las instituciones escolares correspondientes, y más a su necesaria articulación con las otras libertades mencionadas por González del Valle: la libertad de enseñanza, que permite la apertura de centros acordes con un determinado ideario, y la libertad de estudio, que sólo puede asegurase mediante el efectivo ejercicio de la anterior (1984, p. 323). Estas tres libertades, concebibles también como derechos a la educación, a la enseñanza y a la cátedra, mantienen una relación de contención: la libertad de cátedra queda contenida por la libertad de enseñanza, y la libertad de enseñanza queda contenida por la libertad de educación, pudiendo las tres ser definidas con respecto a un concepto de ciudadanía que, como la educación, ponga a la persona en el centro de sus intereses:

- El derecho a la educación es el de disfrutar de las mismas oportunidades de desarrollo personal que el resto de futuros conciudadanos.

- El derecho a la enseñanza es el de ofrecer aquellas oportunidades de desarrollo personal que más se ajusten a las distintas maneras de ejercer la ciudadanía en el marco de un pluralismo moral democrático, a la manera de William James (TALISSE; HESTER, 2004).

- El derecho de cátedra es el de ejercer una función social fundamental con sujeción únicamente a los límites impuestos por la razón científica y la razón jurídica.

Por tanto, la libertad de cátedra, entendida como la posibilidad de plantear y expresar siempre y en todo lugar lo que cada quien considere oportuno, es una quimera; pero entendida en sentido amplio como "posibilidad de expresar las ideas o convicciones que cada profesor asume como propias en relación a la materia objeto de su enseñanza" (STC 217/1992, §2.3), es un verdadero derecho, una cátedra sólo cautiva por las otras libertades constituidas en el mismo acto de decisión colectiva: las de enseñanza y educación.

Exploremos, pues, este primer principio teniendo como guía esta triada de libertades, que se corresponden con otros tantos derechos, y con un número no menor de obligaciones. 


\section{SUBPRINCIPIO DE AJUSTE AXIOLÓGICO}

Lo axiológico, lo relativo a los valores, caracteriza el derecho a la educación, entendido desde un punto de vista general como derecho a ser educado. En una sociedad abierta y democrática, este derecho subjetivo, garantizado por la gratuidad de la educación básica obligatoria, es inseparable de su correlato objetivo, encarnado en forma de expectativa legítima por parte de los miembros de la sociedad que la financia vía impuestos: el derecho a educar. Esta relación triádica entre estado, impuestos y educación es la que justifica la existencia de unos fines cuya función, como dijo Luhmann, es "señalar los rendimientos que el sistema tiene que entregar a su entorno para mantenerse" (en: JOAS, 2013, p. 208). En nuestro caso, "lo que se busca con la educación es un fin, y el valor es el fin al que se tiende. Lo axiológico del fin es lo que lo hace deseable y es la base que orienta todo el proceso educativo" (EXPÓSITO; MARSOLLIER; DIFABIO DE ANGLAT, 2018, p. 4).

Nuestra referencia es la acción, y nuestra finalidad el desarrollo de una capacidad de acción que podemos llamar, con Colapietro (1988; 1989; 2009), "agencia humana" para referirnos a unos seres humanos que, "tal y como son retratados por los pragmatistas clásicos, son actores situados y, como tales, seres que responden creativamente" (COLAPIETRO, 2009, p. 1). En consonancia con lo expuesto en nuestra introducción, esta capacidad es la de formar parte de procesos semióticos en los que participamos como signos: no hay mejor forma de descubrirnos y entrenarnos como agentes que la ofrecida por las situaciones ludomotrices. Este ha sido siempre el mascarón de proa de la nave praxiológica:

\footnotetext{
En la actividad física se solicita la personalidad entera, siempre y cuando el que actúa se sepa agente.

Por lo tanto, es necesario que el profesor de educación física sea conocedor de estas riquezas para hacerlas brotar, por una parte, y para mantenerlas bajo control, por otra. Su papel consiste en hacer que sus alumnos tomen conciencia de su existencia como agentes y de su valor como creadores de acción, poniendo el acento en la participación y rechazando la pasividad. Implicado a fondo en cada una de sus acciones, el niño se reconoce como ser original dotado de posibilidades de acción, con posibilidad de contribuir y responsable de sus respuestas psicomotrices (1959: PARLEBAS, 2017, p. 53).
}

Los fines no sirven para definir qué es la educación física (1967: PARLEBAS, 2017, p. 84), pero de la misma manera que no puede haber una educación física sin finalidades, nuestro proyecto no podría tener otro objetivo general que educar para la acción mediante la acción motriz*, mediante una "realización de conductas motrices" diseñada, facilitada y guiada por motricistas competentes que comprenden los sistemas praxiológicos y su conexión con las distintas dimensiones de la conducta motriz. 


\section{SUBPRINCIPIO DE AJUSTE PRAXIOLÓGICO}

El paso de los fines a los medios es el paso de lo axiológico a lo praxiológico, ya que el derecho a la enseñanza sólo puede ser ejercido desde la responsabilidad de quien es competente para desempeñarlo. Una educación física práxica necesita una teoría praxiológica que concilie la razón de ser del sistema educativo con su forma necesaria de ser: "Esta unión, la de la intención que se hace acto, es la que permite pasar del valor elegido al valor actualizado" (1959: PARLEBAS; 2017, p. 55).

$\mathrm{Ni}$ todos los medios permiten alcanzar todos los fines, ni todas las teorías permiten educar para la acción. Ni cualquier teoría de la acción sirve para el desarrollo de una educación física práxica, ni la imprescindible superación de la perspectiva racionalista y cartesiana de la conducta que trajo aparejada la psicomotricidad de Le Boulch fue suficiente para atender a la socialidad primaria que para Joas caracteriza a la acción humana, junto con la situación y la corporeidad (2013, p. 201 y ss.).

La psico-socio-motricidad, nacida en un entorno de crisis y revolución, es la única salida a la "educación física desmenuzada" (1967: PARLEBAS; 2017, segunda parte) que puede tener en cuenta, de forma unitaria y coherente, las tres finalidades esenciales de toda educación: "conocimiento y dominio de uno mismo, [...] adaptación al medio [...y] aprendizaje de la vida en grupo":

Hay que conseguir integrar esos tres aspectos en una educación física homogénea y unitaria, para lo cual es necesario ser fieles a la idea-fuerza de las ciencias humanas: el hombre es una totalidad inseparable de su medio físico y social (1967: PARLEBAS; 2017, p. 75).

Nos atrevemos a afirmar que sólo los conceptos praxiológicos fundamentales (conducta y acción; juego deportivo y tarea; situación y lógica interna; universal y etnomotricidad, etc.) permiten considerar, comprender, orientar y educar esta totalidad multidimensional e histórica que llamamos persona, de forma homogénea y unitaria:

Un esgrimista, un esquiador o un jugador de fútbol no pueden
ser asimilados, como se ha creído durante tanto tiempo, a
maquinas biológicas cerradas sobre sí mismas: las explicaciones
referidas a las palancas y al gasto energético resultan
insuficientes. Las conductas motrices del practicante son la
manifestación de una personalidad que interactúa con su ambiente
físico y su entorno social. Comprender al individuo que actúa pasa
por el conocimiento del sistema global del que ese individuo forma
parte (p. 445).

No todas las propuestas pedagógicas pueden apelar con igual fuerza al mítico desarrollo integral de la personalidad. 


\section{SUBPRINCIPIO DE AJUSTE METODOLÓGICO}

Como hemos visto, la acción docente es tan libre como lo que permitan el derecho a ser educado para la libertad y el derecho a educar para la libertad. Todas las propuestas pedagógicas se proponen respetar y defender ambos derechos, del mismo modo que todas las propuestas didácticas se consideran capaces de conectar las distintas dimensiones de la intervención motriz -estilo, estrategia y evaluación (MARTÍNEZ-SANTOS, 2019) - y hacer posible la transformación de los efectos deseados* en efectos obtenidos*, mediante la correcta gestión de los efectos esperados* de las tareas* que son llevadas a la práctica.

Esta tercera restricción es, paradójicamente, las más liberadora de todas, ya que reconoce en las teorías un sistema de creencias científicamente adquiridas que orientan las decisiones docentes asegurando una alta probabilidad de acierto. La educación física científica, en tanto que comunidad profesional arraigada en la universidad, obliga tanto como capacita, constriñe tanto como redime, exige tanto como da.

La educación física será científica o no será. Defender este punto de vista no implica dejarse arrastrar por un anticuado positivismo - la sacralización de la cifra y la medida: es una búsqueda de lucidez. Se trata, simplemente, de poner en claro las hipótesis que guían la acción, de organizar experimentos para probar estas hipótesis, de interpretar los resultados obtenidos tanto como sea posible (PARLEBAS, 1971) . $^{5}$

La educación física práxica es una educación física en la que estructura, génesis y motricidad van de la mano (1968-70: PARLEBAS; 2017, tercera parte), tanto a la hora de analizar la ludomotricidad, como a la hora de comprender los fenómenos de aprendizaje, transferencia y adaptación. En este sentido, las nociones hábito, competencia y propósito (COLAPIETRO, V., 2009) del pragmatismo y la semiótica apuntan directamente hacia la posibilidad de provocar en quien aprende la mayor claridad de ideas posible, en forma de hábitos motores y esquemas corporales, claro está.

En caso de existir, desgraciados e indeseados accidentes aparte, la mala praxis docente en educación física sólo se podría llegar a reclamar a quien, por indolencia o ignorancia, no pusiera los medios necesarios para que en cada persona a su cargo se desarrollaran, en su mayor grado de claridad posible, aquellas competencias motrices que nuestra cultura y sociedad, nuestros educadores familiares e institucionales, y nuestro nivel académico y científico, nos dan derecho a esperar.

\footnotetext{
5 La Revue E.P.\& S. en la que Parlebas publicó sus teorías puede consultarse en internet gratuitamente: http://uv2s.cerimes.fr/media/revue-eps/. No están todos sus artículos disponibles, pero merece la pena echarle un vistazo.
} 


\title{
PRINCIPIO DE (IN)COMPATIBILIDAD LÚDICA
}

El principio de cátedra cautiva nos conecta con la dimensión jurídica de la educación física escolar: la educación básica es un ámbito de acción docente creado en forma de ley que regula la actividad de los agentes educativos, cuyos actos están orientados por fines preestablecidos y condicionados por los medios puestos a su disposición. Hablar de libertad de cátedra es, en realidad, otra forma de referirse a la naturaleza creativa (COLAPIETRO, V. M., 2009) de ese tipo de agencia humana que llamamos educar, aunque sea una libertad limitada...

Nuestro segundo principio, el de (in)compatibilidad lúdica, remite a la que podemos denominar dimensión pedagógica. No encontramos ya en el área curricular "educación física", y nos debemos preocupar ahora por la conexión entre los fines educativos encomendados y los medios de enseñanza de que disponemos: las distintas lógicas de acción motriz. John Dewey (1859-1952), cuyas obras siguen siendo fuente de inspiración para los educadores del siglo XXI, fue un pragmatista tardío que elaboró su credo pedagógico alrededor del principio de actividad, a partir de la acción como principio pedagógico:

\begin{abstract}
Creo que la única y verdadera educación viene dada por la estimulación de las capacidades del niño que las demandas de las situaciones sociales en las que se encuentra producen. A través de estas demandas, es impulsado a actuar como miembro de una unidad, a salir de su estrechez de acción y sentimiento iniciales, y a concebirse a sí mismo desde el punto de vista del bienestar del grupo al que pertenece. A partir de las respuestas que otros dan a sus acciones (activities) llega a saber qué significan en términos sociales, cuyo valor se refleja a su vez en ellas $(1897$, p. 3$)$.
\end{abstract}

Dewey, para quien la escuela (primaria) "debe representar la vida presente, una vida tan real y vital para el niño como la que lleva a cabo en el hogar, en el vecindario o en el campo de juego" (p. 7), avanzaría hacia una segunda concepción en la que la acción no es sólo el punto de partida, sino también el punto de llegada, el objetivo mismo de la educación: como apunta Goodman, la educación sería, para Dewey, "la búsqueda de una vida verdaderamente interesante, una vida en la que nos interesamos activamente, más que una vida en la que el interés se ausenta o se pospone" (2007, p. 220).

A nadie sorprenderá saber, por tanto, que Dewey incluyó el juego entre las actividades con potencial educativo: ¿acaso hay actividad más infantil que el juego? Sin embargo, el binomio juego/educación nos hace darnos de bruces con una pequeña paradoja: la de querer (im)poner un tipo de experiencia, la lúdica, ique es libre por definición! Por esta razón hemos decidido formular este principio como el de la (in)compatibilidad lúdica, atemperando nuestro impulso inicial con unos prudentes paréntesis. 
En contadas ocasiones se tiene la oportunidad de disfrutar de propuestas sobre la educación física que provengan de personas ajenas a nuestro campo. Sara Barrena, en su precioso trabajo Pragmatismo y educación. Charles S. Peirce y John Dewey en las aulas, nos regala una de estas ocasiones, junto con un montón de claves para conocer y entender autores e ideas imprescindible. Como dice en la contraportada:

La acción y sus posibles consecuencias, el razonamiento mediante hipótesis -que combina rigor e imaginación-, la valoración positiva del error, el fomento del autocontrol, el desarrollo de hábitos de crecimiento y la búsqueda de un espíritu científico que promueva en los alumnos la investigación y la comunidad constituyen las claves que nos ofrece el pragmatismo para la educación (BARRENA, 2015).

Aplicando el pensamiento pragmatista a la educación física, nos dice: "Toda educación ha de tener por tanto en cuenta la dimensión corporal en conexión con el espíritu, y fomentar a través del juego y del deporte el espíritu lúdico, libre y creativo" (p. 168).

Antes de valorar precipitadamente la doble presencia de lo espiritual en su conclusión, cabe recordar la profundidad de significado que las palabras pueden llegar a asumir en filosofía. En este sentido, resulta gratificante comprobar que en nuestro campo podemos alcanzar, también, altos niveles de sofisticación conceptual y especificidad desde los que reinterpretar afirmaciones vertidas que, por otro lado, también son frecuentes entre nosotros: "el juego permite el desarrollo de la imaginación" (p. 164), "permite desarrollar nuestra capacidad simbólica" (p. 165), "el juego y el deporte son una forma de expresión", "el juego contribuye al desarrollo de valores" (p. 166), "el juego nos enseña a seguir normas" , y "el juego fomenta nuestra libertad":

El juego enseña al niño tanto a imaginar como a seguir unas reglas, dos aspectos esenciales para la creatividad y el crecimiento, pues lo creativo debe serlo dentro de un sistema que tiene unas determinadas reglas y en el que hay que aprender a manejarse. De otro modo, lo supuestamente creativo no sería sino una excentricidad (p. 167).

No anda Sara Barrena desencaminada, y su cita de Aldous Huxley lo certifica: "El deporte puede ser empleado con buenos y malos propósitos. Bien empleado puede enseñar a ser tesonero y valeroso [...]; mal usado, es un estímulo de la vanidad individual y de la vanidad de grupos". Dicho en palabras de Parlebas: "El deporte no es ni nocivo ni virtuoso en sí mismo, aunque puede serlo según el contexto: el deporte será lo que se le haga ser" (1967: 2017, p. 87).

Cualquier situación motriz, juegos deportivos institucionalizados incluidos, tiene un potencial formativo que sólo saldrá a la luz si las conocemos suficiente y pertinentemente. 


\section{SUBPRINCIPIO DE EXPLORACIÓN LUDOMOTRIZ}

Los dominios de acción motriz*, un primer nivel de abstracción operativa de la lógica interna* de las situaciones ludomotrices, son el referente fundamental para pensar los denominados bloques de contenido de nuestra área curricular. Curiosamente, no aparecen como entrada en la primera edición del léxico (1981), aunque la clasificación de Parlebas siempre ha sido un sistema de categorías en el que cada clase se corresponde con un dominio de acción motriz: "campo en el que todas las prácticas corporales se consideran homogéneas con respecto a criterios pertinentes y precisos de acción motriz" (2001, p. 161).

La clasificación de las "situaciones deportivas que se ofrecen al practicante" tuvo lugar en el número 103 de la Revue EP\&S (1970: PARLEBAS; 2017, p. 177 y ss.), que incluía un artículo titulado La educación física: una educación de las conductas de decisión, publicado en el número siguiente al que incluía otro no menos relevante: La afectividad, clave de las conductas motrices (p. 167 y ss.). Desde aquel momento, las nociones incertidumbre*, decisión motriz*, comunicación práxica*, metamotricidad*, principio de acción*, inteligencia motriz*, praxema*, etc., han configurado un modo extremadamente dinámico y avanzado de pensar las situaciones ludomotrices y comprender las conductas motrices.

Si la clave de la acción motriz es el tipo de relación que los agentes establecen con el medio físico y el entorno social, la principal consecuencia de esa relación es la incertidumbre que se les genera a los agentes a la hora de actuar; si la conducta motriz es una manifestación de la personalidad, las decisiones tomadas y las omisiones asumidas por los agentes son signos e interpretaciones de esa relación entre agentes y sistemas ludomotores que llamamos lógica interna* de la situación, del juego o de la actividad.

En definitiva, la presencia o ausencia de incertidumbre generada por la relación con el espacio (I), de compañeros (C) y de adversarios (A) da lugar a las ocho clases de situaciones motrices tan conocidas, los CAIs, referencia fundamental de nuestra educación física:

La distribución de las actividades físicas y deportivas en un determinado número de dominios de acción pertinentes ofrece al motricista una referencia básica para sus decisiones, y constituye la piedra angular de la programación de la educación física. Es vital, en efecto, conseguir un ajuste eficaz de las actividades motrices que se proponen y los objetivos que se persiguen. Esta distribución en dominios de acción motriz no es, por tanto, un mero razonamiento convencional o académica, sino un enfoque fundamental que organiza las situaciones a partir de la comprensión de los efectos esperados y, lo que es mejor aún, de los efectos obtenidos (PARLEBAS; 2001, p. 166). 


\section{SUBPRINCIPIO DE EXPLORACIÓN SEMIOTRIZ}

La clasificación de tareas motrices por dominios permite concebir de forma operativa la educación física básica, aquella que tiene por objetivo dotar a todas las personas de un determinado nivel de competencia en todos los dominios de acción motriz. Sabemos, con todo, que el propio Parlebas (2003) participó en el grupo de trabajo que llegó a proponer al ministro francés de educación del momento una renovación de los contenidos de enseñanza a partir de dominios de acción, aunque finalmente no fue fiel a su palabra ministerial de convertirla en instrucciones oficiales (PARLEBAS, 2006).

Pasados por el tamiz de la institución escolar, "los dominios de acción motriz en la educación física" se convierten en siete, "apoyándonos en las clases de equivalencia del símplex, si bien reestructurándolas según los imperativos prácticos (un número de dominios bastante restringido) y las demandas sociales (éxito de ciertas prácticas)" (PARLEBAS; 2003, p. 38): tres dominios de acción sociomotriz, a saber, pura cooperación ( $\mathrm{C}$ y CI), pura oposición ( $\mathrm{A}$ y $\mathrm{AI}$ ) y colaboración-oposición ( $\mathrm{CA}$ y $\mathrm{CAI}$ ); tres dominios de acción psicomotriz, a saber, acción en medio incierto (I), acción en medio conocido con mejoras objetivas cifradas $(\varnothing)$ y acción en medio conocido con pruebas de valoración subjetiva $(\varnothing)$; y el dominio de acción de las actividades físicas artísticas.

Con todo, esta revisión abre la posibilidad de una reestructuración más profunda. La distinción de dos clases de motricidad (la ludomotriz y la artística, por decirlo de alguna manera) nos recuerda que la semiotricidad es el núcleo duro de la acción motriz. En caso de ser necesario, la reclamación de una competencia motriz, al mismo nivel que el resto de competencias básicas, puede ser defendida con más éxito si se plantea como una competencia semiotriz en la que se pueden distinguir tres grandes vectores, en consonancia con el análisis de la metamotricidad de Parlebas (1979: 2017, p. 36 y ss.):

- Una semiotricidad relacional, de tipo socio-afectivo, "que opera sobre el valor afectivo y relacional que los protagonistas otorgan a sus intercambios motores", incluyendo los paradójicos.

- Una semiotricidad referencial "parecida al lenguaje, en la medida en que transmite un mensaje referido a un contenido ajeno a la motricidad inmediata", con trazas icónicas, indiciarias y simbólicas variables, acompañada de música a veces.

- Una semiotricidad instrumental, no limitada a las situaciones de enfrentamiento "que solicitan una metamotricidad operativa al servicio de comportamientos inequívocos de victoria", que incluya la espacial y hasta su ausencia: aplicada a los procesos de enseñanza-aprendizaje de 
Ios automatismos, la semiotricidad de uno mismo desaparece con la incertidumbre asociada a la impericia, a la falta de competencia.

\section{SUBPRINCIPIO DE EXPLORACIÓN ETNOMOTRIZ}

Una gran parte de los contenidos de la educación física son, en realidad, continentes: grandes continentes de actividades que las agrupan bajo nombres tan evocadores como juego o deporte. La etnomotricidad*, "campo y naturaleza de unas prácticas motrices que son consideradas desde el punto de vista de su relación con la cultura y el medio social en que se han desarrollado", es un tercer nivel de abstracción pertinente para la educación física que completa a los dos anteriores: la lógica interna de los juegos deportivos (ludomotricidad), la competencia motriz general (semiotricidad), y la cultura ludodeportiva (etnomotricidad) conforman una tríada de conceptos tan fundamentales como operativos para el desempeño del oficio de motricista*.

Hace ya años que Parlebas (2008) revisitó las categorías ludodeportivas elementales sobre las que edificó, entre otras, su teoría sociológica (1988), enriqueciendo nuestro conocimiento del ludorama a disposición de la educación física y suscitando nuevos retos. La taxonomía de las actividades ludomotrices se construye por la acumulación de tres rasgos diferenciales: la presencia o ausencia de reglas, competición e institucionalización:

- Los cuasijuegos* son actividades no regladas, informales, carentes de restricciones; son acciones en el sentido más gramatical del término: caminar, nadar, correr, jugar...

- Cuando este jugar está reglado hablamos ya de juego motor. No todos los juegos motores regulan enfrentamientos: los corros y los juegos de palmas, por ejemplo, son meros procedimientos creados mediante consignas. Los juegos motores reglados son los juegos deportivos*.

- Los deportes* son los juegos deportivos institucionalizados*. Aquellos que no lo son, o lo son en grado menor, son los denominados cuasideportes*, los juegos deportivos tradicionales* o de convivencia, los juegos de calle (BORDES; LESAGE; LEVEL, 2013) y los videojuegos.

Las cuatro categorías de situaciones motrices previstas por Parlebas para la docencia están contenidas en este ludorama: los cuasijuegos, los deportes (y cuasideportes), los juegos tradicionales, y las "situaciones didácticas: marcadas por instrucciones, consejos, sugerencias: ejercicios--problema, situaciones adaptadas, ejercicios preparatorios y educativos, ejercicios con orientación variada (relajación, yoga, expresión corporal, eutonía...)" (2001, p. 174). Es evidente que la nueva denominación de los juegos tradicionales (juegos de convivencia) encaja con la semiotricidad relacional antes mencionada, pero 
resulta mas evidente todavía que la presencia de los videojuegos (entendemos que se trata de los denominados exergames) provoca perturbaciones profundas en los limites de la concepción de nuestro objeto de estudio (acción motriz) y nuestro objeto de intervención (la conducta motriz) que debemos analizar con detenimiento.

\section{PRINCIPIO DE EVALUACIÓN PRÁXICA}

Cada tarea escolar, por minúscula que sea, exige una respuesta, y cada respuesta, incluida su ausencia, es un acto interpretable que, mientras completa la interacción educativa en marcha, da pie a infinidad de nuevas interacciones educativas. En cada uno de estos fenómenos de semiosis educativa ilimitada en espiral está presente la valoración. Dado el carácter fundamental de la acción humana en nuestro proyecto, hemos considerado importante destacarlo en este tercer y último principio curricular: el de evaluación práxica.

Tras los fines y los medios, nos toca ahora lidiar con los rendimientos del sistema de los que hablaba Luhmann: los efectos educativos*, consustanciales a cualquier proceso de enseñanza, e imprescindibles a la hora de justificar puestos, proyectos y presupuestos. Para quien resulte excesivo hablar de rendimientos y sistemas, podemos pensar este elemento curricular con ayuda de Ortega y Gasset: "Yo soy yo y mi circunstancia, y si no la salvo a ella no me salvo yo". La evaluación, en definitiva, es el proceso que nos permite saber si hemos sido capaces de adaptarnos a las circunstancias, de ajustarnos a la situación actuando de manera apropiada, calculando incluso el grado de cumplimiento mostrado.

Desde un punto de vista estrictamente praxiológico, los significados asociados a los comportamientos motores de quienes participan en una situación motriz son interpretables en función de la lógica relacional, referencial o instrumental en la que les ha sido dado participar: adhesiones y traiciones; evocaciones y rememoraciones; oportunidades, embates y rechaces. Desde un punto de vista estrictamente evaluativo, todos estos significados primeros son signos interpretables que los conectan con los objetos propios de la intervención y la educación: la inteligencia, la competencia y el autocontrol. Desde un punto de vista estrictamente semiótico, una valoración es siempre un signo interpretante que nos conecta con el objeto de conocimiento a partir de los signos por él provocados.

La misma estructura lógica triádica que nos ha acompañado a lo largo de este ensayo, es la que nos va a dar el pie para el mutis en este tercer y último acto de la segunda parte. Va a ser imposible profundizar en cada uno de los aspectos de la evaluación, aunque no se trata ahora de resolver casos concretos, sino de exponer nuestra manera de pensarlos y apuntar las cuestiones 
pertinentes de la manera más operativa posible: cuál es el efecto real de la práctica motriz, cuáles son los signos que mejor nos informan sobre esa realidad, y cuáles son las líneas de desarrollo educativo que nos permitan dar sentido a un proceso que extiende durante doce años.

Nuestra forma de evaluar es nuestra forma de valorar, son nuestros valores puestos en acción. La evaluación puede ser la clave de la educación física escolar.

\section{SUBPRINCIPIO DE ESPECIFICIDAD MOTRIZ}

Cualquiera que sea, nuestra evaluación manifiesta la creencia de que la intervención motriz deja huella, de que una correcta organización didáctica causa transformaciones socialmente valoradas. Cualquiera que sea la metáfora elegida (AHAMADY; YAGHMAEI; ARAB; MONAJEMI, 2016; GUILHERME; SOUZA DE FREITAS, 2018), en todos los casos hay algo que se produce artísticamente, se fabrica industrialmente o se transporta. Y hasta en los casos en los que se reniega del objeto en defensa del sujeto, lo otro subjetivo no deja de formar parte de una realidad objetiva, de un mundo que puede ser, es y será con independencia de nosotros, y con el que también establecemos una relación triádica.

Por tanto, podemos hablar y considerar un objeto de la intervención motriz que es también una posibilidad, una actualidad y una apertura de relación con la realidad social y material del universo con el que interactúa: ¿qué otro sentido puede tener, si no, decir que los juegos son "una microsociedad" (PARLEBAS; 2017, p. 89, 101, 381) y hasta "un microcosmos" (GUILBAUD; 1964, en: PARLEBAS, 1972, p. 25)? Piaget nos enseñó que la inteligencia, en tanto que apertura al mundo, surge de la acción; nos mostró cómo las conductas motrices permiten la construcción del conocimiento de la realidad de forma cada vez más avanzada; exploró como nadie hasta entonces en qué consiste y cómo se da esa subjetivación en la que

[...] la inteligencia y la creatividad suceden a los espacios de juego, que ya aparecían en la regulación instintiva de la conducta. La intencionalidad prerreflexiva del cuerpo humano no es, pues, una reliquia de la conducta animal a la que supera la pura espiritualidad, sino que es la estructura típica de relación entre organismo y entorno (JOAS, 2013, p. 231).

Como dice Joas, "la instrumentalización del propio cuerpo del actor tiene que verse como resultado de un desarrollo [que] requiere una hipótesis evolutiva para su comprensión", y parece que sólo hay una vía de discusión científica donde se haya abordado este tema: las investigaciones sobre el «esquema corporal» o la «imagen corporal»". Parlebas recuperó la noción esquema corporal* para la segunda educación del léxico de praxiología: 
Representación vivida del propio cuerpo y de sus posibilidades dinámicas de intervención, conectada preventivamente con las características vinculadas al espacio, los instrumentos, el tiempo y las otras personas impuestas por las limitaciones de la acción motriz en cuestión (2001, p. 202).

Sin embargo, como es habitual, esta recuperación vino acompañada de sutiles apuntes, ampliaciones y aclaraciones con respecto a la concepción psicomotricista de esquema corporal como "patrón estándar" que sirve de referencial básico para toda la motricidad y que se completa alrededor de los 1112 años:

El esquema corporal no es una realidad aislable, sino un concepto, una expresión práctica, y así la vamos a emplear. La representación del cuerpo, basada en sensaciones visuales, táctiles, vestibulares, cenestésicas, etc., abarca realidades extremadamente diversas: cada actividad, cada situación motriz requerirá un esquema corporal específico (p.205).

Sabemos que "mediante las reglas del juego y los códigos en vigor que determinan la lógica interna de las prácticas ludodeportivas, los grupos sociales y las instituciones imprimen su sello en el esquema corporal de los practicantes" ( $p$ 206). Emmanuel Fernandes (2014) ha mostrado con maestría que la especialización deportiva es una realidad en los primeros años de escolarización, que las representaciones corporales dibujadas apoyan la afirmación de que "la lógica interna de cada práctica influye, en función de sus características, en la vivencia corporal y en las representaciones que la acompañan. A cada actividad motriz le corresponde un esquema corporal" (PARLEBAS; 2001, p. 205).

Conjugada ya en plural, la noción esquema corporal nos permite referirnos a la continuidad que existe entre el mundo interior y el mundo alrededor, una continuidad que se pone de manifiesto y resulta inteligible, precisamente, en forma de semiotricidad. Sin embargo, no parece tener mucho sentido evaluar esquemas corporales, si es que fuera posible, sino las actuaciones que emergen de la resolución de tareas motrices en las que las finalidades educativas se hacen presentes en forma de competencias motrices.

La competencia motriz parece haberse convertido en un cliché, en una palabra comodín cuya principal aportación es connotativa: sin importar su contenido semántico, lo que más parece importar es el valor que da a quien lo usa. Sin embargo, nos encontramos ante una noción que puede sernos muy útil, entre otras cosas porque su conjugación (ser competente, adquirir o desarrollar competencias, niveles de competencia, etc.) permite identificar y nombrar fenómenos en un nivel de elaboración superior al de los datos brutos de la realidad. En este caso también, Parlebas (2018), valiéndose de las distinción que hace Chomsky entre competencia y actuación (competence and performace), da por buena la definición del consejo francés de programas: "Capacidad para 
movilizar recursos (tanto saberes como saber-hacer y saber-ser) en la realización de una tarea o al hacer frente a una situación". Dicho de otro modo:

Hay que evitar a toda costa el error de reducir la competencia práxica a un inventario de actuaciones concretas, las cuales, por indispensables que sean, solamente sería indicadores de una capacidad más amplia susceptible de producir respuestas inéditas, fértiles en creatividad.

La noción competencia se ha dejado atrapar por una lógica de evaluación de comportamientos que toma sus referencias de criterios reproducibles, normalizados y jerarquizados, como las rúbricas de un examen o los criterios empresariales de calificación. La competencia no debe amordazar al practicante con un catálogo de respuestas predefinidas, sino abrirlo ante un horizonte de posibles novedades. Cualquier competencia va a estar ligada al contenido de las conductas motrices demandadas por las situaciones concretas, y es que el término "competencia" es plástico y de significado variable, en la medida en que se alinea con las conductas motrices particulares a las que se ha elegido dar cuenta. Así, por ejemplo, hablaremos de una "competencia informacional" y de una "competencia relacional", y propondremos la noción "competencia semiotriz" para indicar que, para la persona en acción, se trata de extraer la información pertinente obtenida de los estímulos del entorno. Esta competencia se manifestará tanto en el surf como en el balonmano, en el escalda o el tenis, por medio de procesos de anticipación, empatía y preacción susceptibles de adoptar manifestaciones inesperadas y no preprogramadas durante el entrenamiento.

La competencia es una manera global de situarse y reaccionar ante el contexto circundante, es una relación con el mundo exterior que apela a la inventiva, la confrontación y la coparticipación. Todos estos procesos remiten a la vivencia y a los recursos de la personalidad de un practicante que se expresa por la actualización de sus conductas motrices: nada tiene sentido al margen de las posibilidades de las conductas motrices de la persona que actúa, lo que supone una puesta en acción de sus capacidades orgánicas, por supuesto, pero también de las cognitivas, las afectivas y las relacionales. Y es la realización de esta acción motriz, precisamente, en sus procesos y representaciones, la que está en el corazón de las propuestas de la praxiología motriz (PARLEBAS;2018, p. 93).

La educación física sólo debe evaluar competencias motrices, so pena de renunciar a lo que la caracteriza y diferencia.

\section{SUBPRINCIPIO DE TRANSFERENCIA INTERESPECÍFICA}

La valoración de los efectos obtenidos al final de cada unidad temática debe hacerse mediante las tareas motrices adecuadas, siendo tan importante lo segundo como lo primero: cualquier tarea de evaluación, ya sea mediante observación o medición, debe ser metodológicamente sensible y fiable; y cualquier tarea de evaluación debe movilizar conductas motrices, en aplicación del subprincipio anterior. 
En este momento nos interesa la relación sintomática que los comportamientos motores, las actuaciones, tienen con el objeto que queremos conocer, la competencia motriz. Se trata, pues, de producir signos de forma sostenible, fiable y pertinente: instrumentos de evaluación adaptados a la realidad del aula, sencillos de manejar, que proporcionen datos que se puedan compartir, y que registren los comportamientos que mejor desvelen la lógica interna de la tarea en cuestión. Afortunadamente, nuestra teoría praxiológica nos permite tomar como referencia los elementos que hemos venido empleando hasta ahora (competencia, dominio, principio de acción y trasferencia) para llevar en nuestra caja de herramientas evaluadoras lo estrictamente necesario.

La evaluación de la motricidad instrumental viene determinada por los dominios de acción. Es evidente que siempre podemos tomar las marcas y las victorias como indicadores de competencia: la competición no hace otra cosa que ordenar deportistas por niveles de desempeño, de forma muy eficiente, por cierto. Sin embargo, y sin desdeñar gratuitamente esta opción, la lógica de la escuela no es la lógica del deporte, la lógica del proceso no es la lógica del resultado, aunque, ciertamente, se deba esperar un nivel de competencia mayor en quien vence. A modo de ejemplo, la herramienta de observación y calificación empleada por Dugas (2006) y Parlebas para la investigación sobre la transferencia de aprendizaje puede ser transformada y ajustada, según las necesidades de cada etapa y de cada docente, para valorar el nivel de competencia en los duelos colectivos ludodeportivos. El mismo sistema de categorías comportamentales y codificación de las variables puede ser empleado con las tareas apropiadas para cada nivel escolar (más o menos jugadores, espacio mayor o menor...), y con una coparticipación mayor o menor de los propios estudiantes.

La evaluación de la competencia motriz artística es una cuestión apasionante, semiótica en sentido estricto y controvertida por naturaleza, ya que tampoco escapa al debate sobre el peso que la formación técnica debe tener en la educación de esta semiotricidad referencial:

El ámbito de la expresión corporal, que no está sometida a las obligaciones del resultado cifrado, es ciertamente el que permite desmomificar al individuo en mayor medida, ya que invita a la liberación, a la creación. Es decir: aunque se apoya en lo conocido, invita a no conformarse con reproducir servilmente lo conocido, lo clásico, sumándose a una evolución que sólo es posible gracias a los hechos anteriores. Como ya no se trata de una reproducción mecánica, el niño es solicitado en sus recursos personales, en su espontaneidad, en su imaginación. Los niños van a actuar según sus propias significaciones, y el espacio, por ejemplo, ya no es un área homogénea e impersonal, sino un sitio habitado por el sentido. [...] La expresión corporal puede dar lugar a escenarios que se experimentan mediante intercambios interpersonales insertos en una dinámica de grupo (como en un juego de roles o en un psicodrama) en la que la dimensión estética siempre estará presente (1968: PARLEBAS, 2017, p. 196) . 
Cada dominio de acción motriz exige un tratamiento específico de valoración de sus competencias, lo que nos permite a cambio ser más eficientes y colaborativos en el desarrollo de nuestros recursos didácticos.

\section{SUBPRINCIPIO DE ELABORACIÓN SIMBÓLICA}

"La referencia no es sólo reverencia. Por paradójico que parezca, pensamos que Piaget ningunea la motricidad en sí misma" (1968: PARLEBAS; 2017, p. 163, cursivas en el original), aunque el hecho de que la función semiótica avance hacia lo simbólico, hacia lo lingüístico en gran medida, no impide que la motricidad también crezca en su compañía. ¿Acaso podemos concebir una educación física escolar sin palabras, si los juegos deportivos pueden no ser otra cosa que palabras que juegan (MARTÍNEZ-SANTOS, 2018)? Desde su concepción jurídica hasta su puesta en marcha corporal, pasado por las distintas concreciones pedagógicas y didácticas, la intervención motriz es el reflejo de la acción de infinitos signos corpóreos, pero no motrices.

En este sentido, la educación física puede contribuir de forma significativa al desarrollo de las competencias básicas curriculares, declarativas principalmente, en su lógica de claridad creciente, en el sentido de Peirce. Sin embargo, esta interdisciplinariedad curricular avasalla nuestra área de la misma manera que la profesional o la académica (2014: PARLEBAS, 2017, p. 57-67). La educación física debe luchar continuamente por no perder peso específico en el currículo, a pesar de lo cual es un recurso privilegiado a la hora de transformar la transversalidad de las competencias básicas en proyectos evaluables. ¿Tanto tiempo nos sobra como para poner nuestra especificidad al servicio de objetivos que, en sí mismos y en otras circunstancias, serían del todo loables? Ciencia, arte, literatura y motricidad pueden darse la mano y llegar más lejos juntos que por separado, pero la malquerida educación física debe cuidarse mucho a sí misma en entornos no tan amables como las colonias o los campamentos (1971: PARLEBAS, 2017, p. 227).

El potencial educativo de la acción motriz no acaba en las piscinas o los escenarios, los parques o las canchas de baloncesto, pero su transferencia a los pupitres sólo es posible si invertimos el sentido de la transversalidad y hacemos que el etnoludismo,

Concepción y constatación de que los juegos están en consonancia con su cultura de pertenencia, en particular las características de su lógica interna que ilustran los valores y el simbolismo subyacente a dicha cultura: relaciones de poder, papel de la violencia, imágenes de hombres y mujeres, formas de sociabilidad, contacto con el medio ambiente... (1971: PARLEBAS, 2017, p. 223),

entre en las aulas del resto de miembros del claustro. En la Historia general de las civilizaciones dirigida por Maurice Crouzet, a la sazón inspector general de la 
instrucción púbica de Francia tras la segunda gran guerra, el deporte sólo parece mencionado en dos ocasiones (p. 314 y 318 ) cuando toca hablar del siglo XIX: una obra de dos volúmenes de 915 páginas en edición de bolsillo. De nosotros depende que los ciudadanos del futuro sepan que, para hablar del pasado, del presente y del futuro, hay que hacerlo también a través de sus juegos y deportes.

\section{POR SUS OBRAS LOS CONOCERÉIS}

Cada uno de nuestros principios responde a las tres preguntas fundamentales de la educación, y ha sido desarrollado a partir de un principio más fundamental si cabe: la acción motriz es la punta de lanza de la educación física:

- Al cuestionarnos el valor de la educación, su finalidad, llegamos a la conclusión de que la perspectiva praxiológica no se limita a lo motor, sino que lo atraviesa todo. La educación para la acción requiere un sistema que identifique los distintos ámbitos de decisión, y respete las libertades de acción que les corresponden, empezando por la de cátedra cautiva, que no por estar limitada deja de ser una auténtica libertad de acción.

- Al cuestionarnos los medios de intervención motriz, sus contenidos, llegamos a la conclusión de que los distintos niveles de análisis de las situaciones ludomotrices nos permiten seleccionarlas, adaptarlas y contextualizarlas en tres niveles crecientes de abstracción: las tareas, los dominios y el ludorama. No hemos logrado resolver la paradoja de la (in)compatibilidad lúdica, pero si negociar con ella de forma suficiente.

- Al cuestionarnos los resultados educativos, los efectos, llegamos a la conclusión de que la educación física dispone de recursos suficientes para justificar su presencia en el currículo, rendir cuentas con eficiencia, y entablar un diálogo de igual a igual con el resto de área: somos capaces de valorar cada situación en función de la lógica más relevante y teniendo en cuenta, ahora también, los niveles crecientes de abstracción y elaboración conceptual.

Formulados de forma operativa, estos principios y subprincipios son inmediatamente reconocibles sobre el terreno, por muy lejos que nos hayamos quedado de un currículo completamente desarrollado, como el de Aragón (LARRAZ, 2004). De todos modos, ¿qué otra cosa se podía esperar de una cuadrilla que se deja arrastrar con tanta facilidad por las metáforas y se encomienda a las musas para resolver retos imaginarios?

Ha llegado el momento de reconocer que nos gusta jugar, y que nunca 
dejamos de hacerlo, ni al trabajar ni al investigar. iMenos mal que Dewey dejó escrito que "estar jugando y serio al mismo tiempo es posible, y define la condición mental ideal" (1910; In, BARRENA, 2015, p. 168)! Parece ser que Peirce llegó a sugerir que la máxima pragmática es "una versión actualizada del dicho evangélico 'por sus frutos los conoceréis' (Mt 7, 16)", y que James afirmó que el pragmatismo es "un nuevo nombre para viejas maneras de pensar" (REDONDO; NUBIOLA, 2010, p. 928). Cabe la posibilidad de que nuestro currículo praxiológico sea, también, una forma nueva de llamar a lo viejo, por lo que nos conformaremos con haber evitado el pecado que Peirce jamás toleró y Parlebas jamás cometió, abriendo la puerta a la imaginación y desbloqueando el camino de la investigación.

\section{AGRADECIMIENTOS}

Este trabajo ha sido realizado coincidiendo con una estancia de investigación del primer autor en el Grupo de Estudios Peirceanos del Departamento de Filosofía de la Universidad de Navarra (https://www.unav.es/gep/), dirigido por Jaime Nubiola.

\section{REFERENCIAS}

AHAMADY, S.; YAGHMAEI, M.; ARAB, M.; MONAJEMI, A. Metaphor in education: Hidden but effective. Journal of Medical Education, 15, n. 1, 2016. Disponible en https://www.researchgate.net/publication/332105759.

BARRENA, S. Pragmatismo y educación. Charles S. Peirce y John Dewey en las aulas. Machado: Machado, 2015.

BORDES, P.; LESAGE, T.; LEVEL, M. Les jeux collectifs de rue : résurgences ou recréation ? Staps, 101, n. 3, p. 33-46, 2013. 10.3917/sta.101.0033. Disponible en https://www.cairn.info/revue-staps-2013-3-page-33.htm.

COLAPIETRO, V. Habit, Competence, and Purpose: How to Make the Grades of Clarity Clearer. Transactions of the Charles S. Peirce Society, 45, n. 3, p. 348-377, 2009. 10.2979/tra.2009.45.3.348. Disponible en www.jstor.org/stable/10.2979/tra.2009.45.3.348.

COLAPIETRO, V. M. Human agency: The habits of our being. The Southern Journal of Philosophy, 26, n. 2, p. 153-168, 1988. Disponible en https://doi.org/10.1111/j.20416962.1988.tb00448.x.

COLAPIETRO, V. M. Peirce's approach to the self. A semiotic perspective on human subjectivity. Albany: State University of New York Press, 1989.

COLAPIETRO, V. M. A revised portrait of human agency. European Journal of Pragmatism and American Philosophy, I, n. 1-2, p. 1-25, 2009. https://doi.org/10.4000/ejpap.961. 
DEWEY, J. My pedagogic creed. New York: E.I. Kellog \& Co., 1897. Disponible en https://archive.org/details/mypedagogiccree00dewegoog/page/n2/mode/2up.

DUGAS, E. La evaluación de las conductas motrices en los juegos colectivos: presentación de un instrumento científico aplicado a la educación física. Apunts, n. 83, p. 61-69, 2006. Disponible en https://www.revista-

apunts.com/apunts/articulos//83/es/083 061-069ES.pdf.

DURING, B. La crisis de las pedagogías corporales. Málaga: Unisport, 1992.

EXPÓSITO, C. D.; MARSOLLIER, R. G.; DIFABIO DE ANGLAT, H. Los valores en educación para una educación sin valores. Revista Dilemas Contemporáneos: Educación, Política y Valores, V, n. 2.38, p. 1-27, 2018. Disponible en https://dilemascontemporaneoseducacionpoliticayvalores.com/ files/2000037367c0f57cec2/18.1.38\%20Los\%20valores\%20en\%20educación\%20para\%20una\%20educa ción\%20sin\%20valores.pdf.

FERNANDES, E. Sport, Représentation du corps et Socialisation: Vers un décryptage d'un monde de symboles: comment observer ce que nos enfants apprennent? Éditions universitaires européennes, 2014.

GOFFMAN, E. La presentación de la persona en la vida cotidiana. Buenos Aires: Amorrortu editores, 1997.

GONZÁLEZ DEL VALLE, J. M. Libertad de cátedra y libertad de enseñanza en la legislación española. Revista Persona y Derecho, n. 8, p. 313-327, 1981. Disponible en https://www.unav.edu/publicaciones/revistas/index.php/persona-yderecho/article/viewFile/32708/28080.

GoODMAN, R. B. Two genealogies of action in prgmatism. Cognitio, 8, n. 2, p. 213-222, 2007. Disponible en https://revistas.pucsp.br/cognitiofilosofia/article/view/12227/9494.

GUILBAUD, G. T. Théorie des jeux. In: ALLEAU, R. (Ed.). Dictionnaire des jeux. Paris: Tchou, 1964. p. 254-270.

GUILHERME, A.; SOUZA DE FREITAS, A. L. Discussing education by means of metaphors. Educational Philosophy and Theory, 50, n. 10, p. 947-956, 2018/08/24 2018. 10.1080/00131857.2016.1198250. Disponible en https://doi.org/10.1080/00131857.2016.1198250.

JOAS, H. La creatividad de la acción. Madrid: CIS, 2013.

LARRAZ, A. Los dominios de acción motriz como base de los diseños curriculares en educación física: el caso de la Comunidad de Aragón en educación primaria. In: LAGARDERA, F. e LAVEGA, P. (Ed.). La ciencia de la acción motriz. Lérida: Servei de Publicacions de la Universitat de Lleida, 2004. p. 203-226.

MANNING, P. Drama as Life: The significance of Goffman's changing use of the theatrical metaphor. Sociological Theory, 9, n. 1, p. 70-86, 1991. 10.2307/201874. Disponible en www.jstor.org/stable/201874.

MARTÍNEZ-SANTOS, R. ¿Juegos de palabras o palabras que juegan? Sobre la naturaleza jurídica de los juegos deportivos. Cultura, Ciencia y Deporte, 13, n. 38, p. 183-194, 2018. 10.12800/ccd.v13i38.1074. Disponible en https://ccd.ucam.edu/index.php/revista/article/view/1074/424.

MARTÍNEZ-SANTOS, R. La intervención motriz como proceso comunicativo. Innovación, 
tradición y formación de formadores. In: ALONSO, S. ;ROMERO, J. M., et al (Ed.). Investigación, Innovación docente y TIC. Nuevos horizontes educativos. Madrid: Dykinson, 2019. p. 1472-1483.

MARTÍNEZ-SANTOS, R. Signos, metáforas y educación física: conectando Peirce y Parlebas mediante la acción motriz. Conexões : educação física, esporte e saude, v. 18, 2020. En prensa.

NANDO, J.; SANZ, J. R. La autonomía del profesorado no universitario: ell derecho a la libertad de cátedra. Profesorado. Revista de currículum y formación del profesorado, 3, 23, p. 517-534, 2019.

PARLEBAS, P. Pour une épistemologie de l'éducation physique. Revue EP\&S, n. 110, p. 15-22, 1971. Disponible en http://uv2s.cerimes.fr/media/revueeps/media/articles/pdf/70110-15.pdf.

PARLEBAS, P. Jeux sportifs et sociomotricité. Revue EP\&S, n. 114, p. 17-25, 1972.

PARLEBAS, P. Elementos de sociología del deporte. Málaga: Junta de Andalucía: Universidad Internacional Deportiva, 1988. 251 p. p. 84-86826-11-x.

PARLEBAS, P. Juegos, deporte y sociedad. Léxico de praxiología motriz. Barcelona: Paidotribo, 2001. 84-8019-550-9.

PARLEBAS, P. Un nuevo paradigma en educación física: los dominios de acción motriz. In: FUENTES, J. P. e BELLIDO, M. (Ed.). Primer Congreso Europeo de Educación Física FIEP 2003. Cáceres (España): Diputación de Cáceres, 2003. p. 27-42.

PARLEBAS, P. L'action motrice, fer de lance de l'éducation physique. Les cahiers de EPS, 34, p. 5-10, 2006. Disponible en http://www.educ-

revues.fr/CEPS/AffichageDocument.aspx?iddoc $=38460$.

PARLEBAS, P. Un ludorama exubérant. In: LOBJOIAS, D. (Ed.). Jeux traditionnels et populaires de Picardie et du Nord-Pas-de-Calais, 2008.

PARLEBAS, P. La aventura praxiológica. Ciencia, acción y educación física. Málaga: Junta de Andalucía, 2017.

PARLEBAS, P. Une pédagogie des competences motrices. Acción motriz, n. 20, p. 89-96, 2018. Disponible en http://www.accionmotriz.com/documentos/revistas/articulos/20 8.pdf.

REDONDO, I.; NUBIOLA, J. Pragmatismo. Diccionario de filosofía. GONZÁLEZ, A. L. Pamplona: Eunsa: 927-931 p. 2010.

ROBLES, G. Las reglas del derecho y las reglas de los juegos. Palma de Mallorca: Universidad de Palma de Mallorca, 1984.

TALISSE, R. B.; HESTER, D. M. On James. Canada: Thomsom Wadswoth, 2004. v. Wadsworth Philosophers Series).

ZORO, J. Images de 150 ans d'EPS: l'éducation physique et sportive à l'école, en France. Croissy: Amicale EPS, 2002. 